\title{
Bacteriology of the conjunctiva in pre-cataract surgery patients with occluded nasolacrimal ducts and the operation outcomes in Japanese patients
}

Yuko Hayashi ${ }^{1}$, Takeshi Miyamoto ${ }^{2^{*}}$ D, Shuko Fujita ${ }^{1}$, Katsuo Tomoyose ${ }^{1}$, Nobuyuki Ishikawa', Masahide Kokado ${ }^{1}$, Takayoshi Sumioka', Yuka Okada ${ }^{1}$ and Shizuya Saika ${ }^{1}$

\begin{abstract}
Background: Contamination of the conjunctiva in association with nasolacrimal duct obstruction is by all accounts a risk factor for infectious endophthalmitis post-cataract surgery.

Methods: All patients who underwent cataract day surgery routinely received nasolacrimal duct syringing with normal saline at the Wakayama Medical University Hospital, Japan, from 2011 to 2013. The microorganisms isolated from conjunctival swab samples of patients with occluded nasolacrimal ducts and their susceptibility to antibiotics, as well as the operation outcomes in all the patients were retrospectively investigated.

Results: Nasolacrimal duct obstruction was observed in 125 eyes of 90 patients $(3.3 \%$; 42 eyes of 30 male individuals, and 83 eyes of 60 female individuals) from a total of 3754 eyes of 2384 patients by using irrigation samples of nasolacrimal ducts. The mean age of the subjects with duct obstruction was $79 \pm 8.5$ years.. In bacterial cultures of swabs from these 125 individuals, microbial growth was detected in 56 samples (i.e. 44.8\%). Coagulase-negative Staphylococcus was detected in 28 eyes, and Corynebacterium species was detected in 17 eyes. Staphylococcus aureus, excluding methicillin-resistant $S$. aureus was detected in seven eyes with nasolacrimal duct obstruction. Methicillin-resistant $S$. aureus was isolated in two eyes with nasolacrimal duct obstruction. Each case was treated with topical antibiotics based on the results of antibiotic sensitivity tests. After culturing of cotton swab samples from the conjunctiva, and using direct micrography of bacteria every 2 or 3 days after starting treatment, and once the results were negative (consecutively tested three times), the patients received cataract surgery. In the current case series, bacteria were not detected in conjunctival swabs obtained consecutively three times for 3 weeks after starting topical antibiotics in 118 eyes from 125 eyes (94.4\%), and later in the remaining patients. No patient required dacryocystorhinostomy to eliminate bacterial contamination in the conjunctiva following topical antibiotic therapy. No patient developed infectious endophthalmitis at least 1-year post-cataract surgery.
\end{abstract}

Conclusions: All the patients receiving cataract day surgery underwent the operation after the elimination of conjunctival microorganism contamination in association with nasolacrimal duct obstruction by using appropriate topical antibiotics.

Keywords: Nasolacrimal duct obstruction, Conjunctiva, Bacterium, Cataract surgery

\footnotetext{
* Correspondence: tmiyam@wakayama-med.ac.jp

${ }^{2}$ Department of Ophthalmology, Wakayama Medical University Kihoku

Hospital, 219 Myoji, Katsuragi-cho, Itogun, Wakayama 649-7113, Japan

Full list of author information is available at the end of the article
} 


\section{Background}

Infectious endophthalmitis occurs in $0.04-0.075 \%$ of patients following cataract surgery [1-3]. Colonies of endogenous normal bacterial flora are the main source of bacterial contamination in the anterior chamber of the eye. Staphylococci, Enterococci, or Gram-negative bacilli could cause poor visual prognoses postinfection, although the incidence is reportedly low [4-7]. Gram negative bacteria reportedly constitute an increasing proportion of the bacteria found in chronic dacryocystitis and they may be a reservoir for postoperative intraocular infection [8]. It was suggested that these bacteria account for higher rates of nasolacrimal duct obstruction among patients who developed infectious endophthalmitis after cataract surgery [9-11]. Furthermore, these studies indicated that nasolacrimal duct obstruction might cause lacrimal sac and conjunctival bacterial contamination even in the absence of dacryocystitis. Such an occurrence could also be a intraocular infection risk factor subsequent to either trauma or surgery.

It is beneficial, therefore, to test for the presence of nasolacrimal duct obstruction prior to cataract surgery to prevent postoperative bacterial infection in the eye. We routinely perform syringing of the nasolacrimal duct in pre-cataract surgery patients. Furthermore, in all the patients having nasolacrimal duct obstruction regardless of the presence or absence of dacryocystitis we examine both their washings and conjunctival swab samples for bacteriological growth. The susceptibilities of the bacteria isolated from the conjunctival swab samples of patients with occluded nasolacrimal duct, to antibiotics, were determined to eliminate conjunctival bacterial contamination. In the current retrospective study, none of the patients received surgical treatment i.e. tubing or dacryocystorhinostomy, for nasolacrimal duct obstruction at our

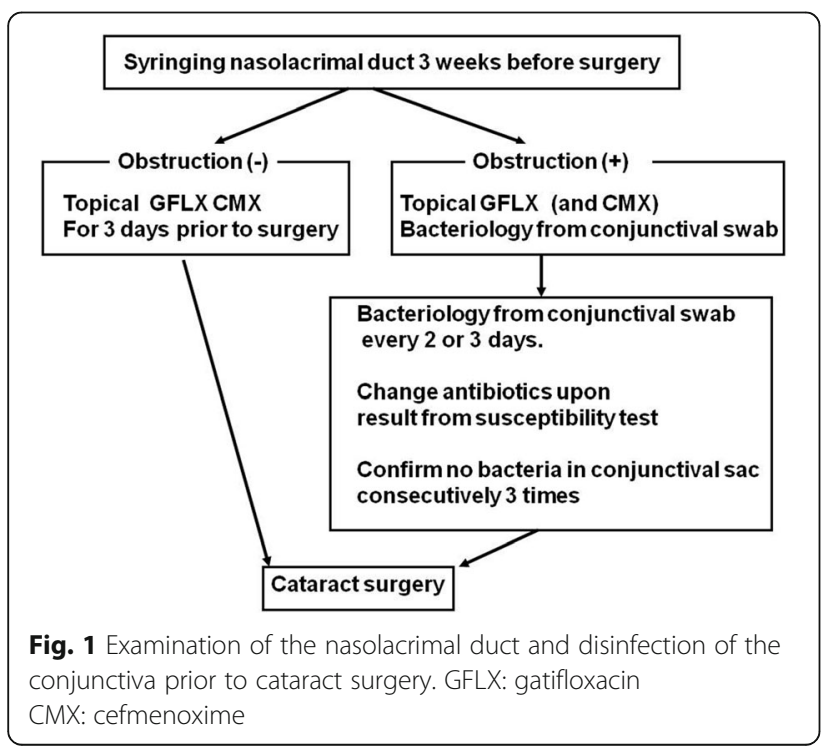

institution to eliminate bacterial contamination in conjunctiva. The present study retrospectively summarizes our results and the outcomes of cataract surgery during 3 years (2011-2013) along with those abovementioned cases receiving pre-surgical antibiotic treatment. As a result of being given suitable topical antibiotics prior to nasolacrimal duct obstruction surgery, even though those individuals had positive bacterial cultures, there were no cases of postoperative endophthalmitis.

\section{Methods \\ Patients}

This retrospective study was approved by the institutional committee for clinical research All the patients undergoing day surgery for cataract (3754 eyes of 2384 patients) received syringing (irrigation) of the nasolacrimal duct with normal saline at Wakayama Medical University Hospital, Wakayama, Japan, 3 weeks prior to the operation due date, for a 3-year period from 2011 to 2013. Bacteriological examinations and/or antibiotic susceptibility testing of samples isolated from conjunctival swabs were performed in patients with obstruction of the duct prior to surgery. Bacteria-positive patients received further examination of conjunctival swab samples at every 2 or 3 days, along with topical administration of antibiotics that were effective for the detected microorganism (Fig. 1). The patients underwent cataract surgery after obtaining negative results for bacterial infection consecutively for three times. Phacoemulsification and aspiration of the crystallin lens with implantation of an intraocular lens (IOL) was performed in 3707 eyes (98.7\%) and planned extracapsular cataract extraction with IOL implantation was performed in 47 eyes (1.3\%). No patient developed infectious endophthalmitis post-cataract surgery.

\section{Bacteriological examinations}

Conjunctival swab samples were obtained from patients with nasolacrimal duct obstruction by using cotton swabs under local anesthesia with topical lidocaine. On the same day, bacteriological culturing (blood agar medium, chocolate agar medium, MacConkey's medium, Colombian nutrient medium, and Candida medium) was conducted. Each culture was incubated in $35{ }^{\circ} \mathrm{C}$ for $16-20 \mathrm{~h}$. Anaerobic culturing was not performed. The minimum inhibitory concentration was classified as "S" (susceptible) or " $R$ " (resistant) by employing the criteria developed by the Clinical and Laboratory Institute.

All patients with nasolacrimal duct obstruction had received topical gatifloxacin $0.3 \%$ (GFLX) before receiving bacteriological examinations as described above. Patients with positive bacterial cultures received further administration of topical cefmenoxime hydrochloride0.5\% (CMX) when the detected bacteria were susceptible to these antibiotics. All patients with detected microorganisms by 
culture examination after detecting nasolacrimal duct obstruction received further conjunctival swab examinations every 2 or 3 days along with topical antibiotics as necessary. Cataract surgery was performed after obtaining no bacterial positive results from the conjunctival swab cultures consecutively for three times.

Prior to the surgery, the eyelid skin and conjunctival sac were disinfected with povidone-iodine. The patients received intravenous sodium piperacillin during or immediately after the surgery. The affected eye received levofloxacin (LVFX) ointment after the operation. From the next day, the patients received topical GFLX for 1-3 months and oral cefcapene pivoxil hydrochloride for 4 days.

\section{Results}

\section{The incidence of nasolacrimal duct obstruction}

Nasolacrimal duct obstruction was observed in 125 eyes of 90 patients $(3.3 \%$; 42 eyes of 30 male individuals, and 83 eyes of 30 female individuals) in a total of 3754 eyes of 2384 patients. The mean age of the subjects with duct obstruction was $79 \pm 8.5$ years.

\section{Bacteriological examinations of conjunctiva with nasolacrimal duct obstruction}

Bacteriological cultures detected microbial growth from the swab samples of 56 of 125 subjects from 90 patients at the initial bacterial culturing (44.8\%). Percentages for Gram-positive cocci, Gram-positive rods, Gram-negative bacilli, and fungi were $64,24,11$, and $1 \%$, respectively (Table 1). Among 125 eyes with positive results for bacteria or fungi, coagulase-negative Staphylococcus (CNS), Corynebacterium species, or $S$. aureus were detected in 28, 17, and seven eyes, respectively. Methicillin-resistant $S$. aureus (MRSA) was observed in two of seven eyes with $S$. aureus.

Table 1 Bacteria and fungus detected at the first conjunctival swab culture in patients with nasolacrimal duct obstruction

\begin{tabular}{lll}
\hline Gram-positive cocci & Coagulase-negative Staphylococci & 28 \\
& Staphylococcus aureus (including & 7 \\
& MRSA in 2 eyes) & \\
& Enterococcus facalis & 3 \\
& Streptococcus speices & 6 \\
Gram-positive bacilli & Corynebacterium species & 17 \\
Gram-negative bacilli & Enterobacter & 3 \\
& Moruganella & 2 \\
& Klebsiella & 2 \\
Fungus & Proteus & 1 \\
\hline
\end{tabular}

MRSA methicillin-resistant Staphylococcus aureus

\section{Bacterial results following antibiotic treatment prior to surgery}

As shown in Table 2, detected microorganisms exhibited resistance to antibiotics. The majority of the bacteria detected were found to be resistant to LVFX, cefazolin (CEZ), and/or gentamycin.

Table 3 includes all the cases in the current series that required additional topical administration of antibiotic eye drops besides the new quinolone drugs and cephem antibiotics. Conjunctival bacterial contamination was successfully eliminated by administration of topical dibeka$\operatorname{cin} 0.3 \%(\mathrm{DKB})$ or arbekacin $0.5 \%(\mathrm{ABK})$ sulfate in case 1 or in cases 2 and 3, respectively. In three eyes of two patients, MRSA was detected at the second or later examination, but not at the first examination. MRSA detected in this case series exhibited resistance to LVFX and CEZ, but was sensitive to vancomycin(VCM). In case 7 , Corynebacterium appeared after Gram-negative bacilli were eliminated with topical GFLX0.3\%. Because this occurred during topical quinolone drug administration, the Corynebacterium was eliminated with topical tobramycin $0.3 \%(\mathrm{TOB})$. In the current case series, no microorganisms were detected in conjunctival swabs consecutively for three times at 3 weeks after starting topical antibiotics in 118 eyes of 125 eyes (94.4\%). Durations of more than 3 weeks were required before no bacteria were detected in swab cultures from conjunctiva taken consecutively for three times in cases 4-7 (Table 3).

Table 2 Sensitivity of detected microorganisms and their resistance to each antibiotic

\begin{tabular}{|c|c|c|c|}
\hline Bacterium species & Antibiotic & Resistant & Sensitive \\
\hline \multirow[t]{3}{*}{ Coagulase-negative Staphylococci } & LVFX & 5 & 17 \\
\hline & CEZ & 5 & 15 \\
\hline & GM & 4 & 15 \\
\hline \multirow[t]{3}{*}{ MSSA } & LVFX & 1 & 5 \\
\hline & CEZ & 0 & 6 \\
\hline & GM & 1 & 5 \\
\hline \multirow[t]{3}{*}{ MRSA } & LVFX & 3 & 0 \\
\hline & CEZ & 3 & 0 \\
\hline & GM & 2 & 1 \\
\hline \multirow[t]{2}{*}{ Streptococcus species } & LVFX & 1 & 2 \\
\hline & CEZ & 1 & 1 \\
\hline \multirow[t]{2}{*}{ Corynebacterium species } & LVFX & 3 & 2 \\
\hline & CEZ & 0 & 4 \\
\hline \multirow[t]{3}{*}{ Gram-negative bacilli } & LVFX & 1 & 7 \\
\hline & CEZ & 3 & 5 \\
\hline & GM & 1 & 7 \\
\hline
\end{tabular}

MSSA methicillin-susceptible Staphylococcus aureus

MRSA methicillin-resistant Staphylococcus aureus

The majority of the bacteria were found to be resistant to levofloxacin (LVFX), cefazolin (CEZ), or gentamycin (GM) 
Table 3 Cases that required administration of additional topical antibiotic eye drops in addition to quinolone and cephem drugs to achieve a negative result for bacterial infection

\begin{tabular}{|c|c|c|c|}
\hline $\begin{array}{l}\text { Case } \\
\#\end{array}$ & Bacterium species & Sensitivity & $\begin{array}{l}\text { Additional } \\
\text { antibiotic }\end{array}$ \\
\hline 1 & CNS & $\begin{array}{l}\text { LVFX (R) CEZ (R) GM (R) ABK } \\
(S)\end{array}$ & DKB \\
\hline 2 & CNS & $\begin{array}{l}\text { LVFX (R) CEZ (R) GM (R) ABK } \\
(S)\end{array}$ & ABK \\
\hline 3 & CNS & $\begin{array}{l}\text { LVFX (R) CEZ (R) GM (R) ABK } \\
(\mathrm{S})\end{array}$ & ABK \\
\hline 4 & MRSA & $\begin{array}{l}\text { LVFX (R) CEZ (R) GM (R) VCM } \\
(\mathrm{S})\end{array}$ & VCM \\
\hline 5 & MRSA & $\begin{array}{l}\text { LVFX (R) CEZ (R) GM (R) VCM } \\
(\mathrm{S})\end{array}$ & VCM \\
\hline \multirow[t]{2}{*}{6} & $\begin{array}{l}\text { MRSA Gram-negative } \\
\text { bacilli }\end{array}$ & $\begin{array}{l}\text { LVFX (R) CEZ (R) GM (R) VCM } \\
(\mathrm{S})\end{array}$ & VCM \\
\hline & & LVFX (S) CEZ (S) GM (S) & \\
\hline 7 & $\begin{array}{l}\text { Corynebacterium Spp. } \\
\text { Gram- negative bacilli }\end{array}$ & LVFX (S) CEZ (S) GM (S) & TOB \\
\hline
\end{tabular}

CNS coagulase-negative Staphylococci

MSSA methicillin-susceptible Staphylococcus aureus

MRSA methicillin-resistant Staphylococcus aureus

DKB: dibekacin0.3\%; ABK; arbekacin0.5\%; VCM; vancomycin; TOB: tobramycin

\section{Discussion}

Obstruction of the nasolacrimal duct causes retention of tears in the lacrimal and conjunctival sac, leading to the acceleration of growth of the microorganisms in the accumulated tears. The likelihood of post-cataract surgery infectious endophthalmitis is much higher in patients whose nasolacrimal ducts are obstructed $[9,10]$. This association suggests that this condition could be a risk factor for intraocular infection subsequent to either trauma or surgery. In addition, $82 \%$ of the bacteria detected from eyes with postoperative infectious endophthalmitis were reportedly genetically identical to those in the normal conjunctival flora [11]. There is no published guideline available in Japan describing a recommended strategy for treating patients with nasolacrimal duct obstruction and have contaminated conjunctivae prior to intraocular surgery. Therefore, examination of the nasolacrimal duct by syringing and bacteriological examinations in eyes with obstruction of the duct should be performed prior to intraocular surgeries according to our protocol.

A previous study found the incidence of adult nasolacrimal duct obstruction to be $3.1 \%$. Anatomical differences may account for the difference in the incidence of obstruction between male and female individuals [12]. Microorganisms were detected in $44.8 \%$ of the eyes with nasolacrimal duct obstruction. We did not perform anaerobic culturing, and this could account for the difference in the detection rate (30-80\%) between our study and the previous studies [13-16].

CNS was the most prevalent finding, and therefore in the current case series, Corynebacterium and S. aureus were detected. This result is similar to that of a previous study, although we did not perform anaerobic culturing and could not detect Propionibacterium acnes [8]. CNS is the bacterial type most frequently observed in postcataract surgery infectious endophthalmitis [4]. Obstruction of the nasolacrimal duct reportedly increases the percentage of Gram-negative bacilli in the conjunctiva in non-Japanese subjects $[8,16]$. Bacteriology of conjunctiva in healthy Japanese subjects without nasolacrimal duct obstruction was also reported [17, 18]. Suto et al. described bacterial presence without screening for nasolacrimal duct obstruction in pre-cataract surgery patients [14]. Thus this data is not applicable to this study. Hoshi et al. reported an increased incidence of Gram-negative bacilli in Japanese subjects as compared with healthy subjects [17]. Moreover, Omatsu et al. reported that the incidence of Gram-negative bacilli was 3.2\% [18]. In the current study, $6.4 \%$ of the bacilli were Gram-negative in the conjunctiva among all subjects, which is indicative of a higher incidence than previously reported. As Gram-negative bacilli are one of the notable bacterial groups that are post-cataract surgery pathogens causing endophthalmitis, these findings support the notion that nasolacrimal duct obstruction could be a risk factor of this infection.

Although Corynebacterium species is one of the most frequently detected bacterial species in normal bacterial flora in human conjunctiva, it was reported that it causes infection in corneas treated with topical corticosteroid therapy after penetrating keratoplasty [19]. Postoperative infectious endophthalmitis is quite rare, presumably because the aqueous humor contains low levels of lipid components that are required for Corynebacterium species to grow. Corynebacterium in the conjunctiva is sensitive to cephem antibiotics and aminoglycosides, while the bacterium is frequently resistant to new quinolones [6]. We successfully eliminated Corynebacterium that newly appeared after the elimination of Gram-negative bacilli by adding the aminoglycoside in case 7 . The phenomenon observed in case 7 was considered to be a form of microbial substitution.

\section{Conclusions}

In summary, nasolacrimal duct obstruction was observed in 125 eyes of 90 patients $(3.3 \%$; 42 eyes of 30 male individuals, and 83 eyes of 60 female individuals) from a total of 3754 eyes of 2384 patients by using samples from syringing of the nasolacrimal duct with normal saline. Each case with positive microorganisms cultured from conjunctival swabs was treated with topical antibiotics based on the results of susceptibility tests. After results from culturing of cotton swab samples from the conjunctiva, and by direct micrography for bacteria every 2 or 3 days (consecutively for three times) were negative, the patients 
received cataract surgery. Normalization of bacterial flora in the conjunctiva with nasolacrimal duct obstruction reportedly requires $4-5$ weeks after dacryocystorhinostomy [20]. Another report showed that dacryocystorhinostomy decreased the percentage of conjunctival sac Gram positive cases from $82 \%$ to $36 \%$ [21] We successfully eliminated bacterial contamination of the conjunctiva in $94.4 \%$ of the patients with nasolacrimal duct obstruction at 3 weeks before cataract surgery, and eventually also eliminated bacterial contamination in all the other cases. In our series, no patient required tubing or dacryocystorhinostomy to eliminate bacterial contamination in the conjunctiva following topical antibiotic therapy. No patient developed infectious endophthalmitis for at least 1-year post-cataract surgery who received topical antibiotic treatment, although previous reports already suggested that nasolacrimal duct obstruction is a potential risk factor of post-cataract surgery infectious endopthalmitis.

\section{Abbreviations}

ABK: Arbekacin; CEZ: Cefazolin; CMX: Cefmenoxime; CNS: Coagulase-negative Staphylococcus; DKB: Dibekacin; GFLX: Gatifloxacin; IOL: Intraocular lens; LVFX: Levofloxacin; MRSA: methicillin-resistant Staphylococcus aureus; MSSA: Methicillin-susceptible Staphylococcus aureus; TOB: Tobramycin; VCM: Vancomycin

\section{Acknowledgments}

The authors thank Dr. Peter S. Reinach for editing English.

\section{Funding}

This study was supported by the Department of Ophthalmology, Wakayama Medical University.

\section{Availability of data and materials}

The datasets produced during and/or analyzed during the current study available from the corresponding author on reasonable request.

\section{Authors' contributions}

All authors participated in obtaining the results as well as in writing and revising the manuscript. Specific additional contributions include: YH, SF, KT, $\mathrm{NI}, \mathrm{MK}$, and TS participated in the examination of the syringed nasolacrimal duct samples and in taking the conjunctival swab samples from the patients. TM, YO, and SS participated in the interpretation of the results. All authors read and approved the final manuscript.

\section{Competing interest}

The authors declare that they have no competing interests and no financial competing interests.

\section{Consent for publication}

Not applicable (no identifying patient data).

\section{Ethics approval and consent to participate}

All study participants gave oral informed consent for bacterial examination prior to their cataract surgeries. This project adhered to tenets of the Declaration of Helsinki. The protocol of the study was approval by IRB of Wakayama Medical University (\#1707). The title of the project approved is "A retrospective study on commensal bacteria in the conjunctiva of pre-cataract surgery patients with nasolacrimal duct obstruction". Each patient with nasolacrimal duct obstruction received explanation about the necessity and usefulness of conjunctival swab sampling. Consent by each of them was indicated on his (her) medical record. We posted a poster which listed study contents on the clinic to get the common knowledge about the purpose of the study according to the instructions of the university authorities.

\section{Author details}

'Department of Ophthalmology, Wakayama Medical University, 811-1 Kimiidera, Wakayama 641-0012, Japan. ${ }^{2}$ Department of Ophthalmology, Wakayama Medical University Kihoku Hospital, 219 Myoji, Katsuragi-cho, Itogun, Wakayama 649-7113, Japan.

Received: 10 August 2016 Accepted: 10 February 2017

Published online: 20 February 2017

\section{References}

1. Wong TY, Chee SP. The epidemiology of acute endophthalmitis after cataract surgery in an Asian population. Ophthalmology. 2004;111:699-705.

2. Lalitha P, Rajagopalan J, Prakash K, Ramasamy K, Prjana NV, Srinivasan M. Post-cataract endophthalmitis in South India: incidence and outcome. Ophthalmology. 2005;112:1884-9.

3. Miller JJ, Scot IU, Flynn Jr HW, Smiddy WE, Newton J, Miller D. Acute onset endophthalmitis after cataract surgery (2000-04) incidence, clinical settings and visual acuity outcomes, after treatment. Am J Ophthalmol. 2005;139:983-7.

4. Peyman GA. Endophthalmitis diagnosis and management 102-107. London and New York: Taylor \& Fransis; 2004

5. Major Jr JC, Engelbert M, Flynn Jr HW, Miller D, Smiddy WE, Davis JL. Staphylococcus aureus endophthalmitis: antibiotic susceptibilities, methicillin resistance, and clinical outcomes. Am J Ophthalmol. 2010;149:278-83.

6. Scott IU, Loo RH, Flynn Jr HW, Miller D. Endophthalmitis caused by enterococcus feacalis: antibiotic selection and treatment outcomes. Ophthalmology. 2003:110:1573-7.

7. Schmidt ME, Smith MA, Levy CS. Endophthalmitis caused by unusual Gramnegative bacilli: three case reports and review. Clin Infect Dis. 1993;17:686-90.

8. Hartikainen J, Lehtonen OP, Saari KM. Bacteriology of lacrimal duct obstruction in adults. Br J Ophthalmol. 1997;81(1):37-40.

9. Kam JK, Cheng NM, Allen PJ, Brooks AM. Nasolacrimal duct screening to minimize post-cataract surgery endophthalmitis. Clin Experiment Ophthalmol. 2014:42:447-51.

10. Lopez PF, Beldavs RA, al-Ghamdi S, Wilson LA, Wojno TH, Sternberg Jr P, Aaberg TM, Lambert HM. Pneumococcal endophthalmitis associated with nasolacrimal obstruction. Am J Ophthalmol. 1993;116:56-62.

11. Speaker MG, Milch FA, Shah MK, Eisner W, Kreiswirth BN. Role of external bacterial flora in the pathogenesis of acute postoperative endophthalmitis. Ophthalmology. 1991;98:639-49.

12. Shigeta K, Takegoshi H, Kikuchi S. Sex and age differences in the bony nasolacrimal canal. Arch Ophthalmol. 2007;125:1667-81.

13. Hara J, Yasuda F, Higashitsutsumi M. Preoperative disinfection of the conjunctival sac in cataract surgery. Ophthalmologica. 1997;211 suppl 1:62-7.

14. Suto C, Morinaga M, Yagi M, Tsuji C, Toshida H. Conjunctival sac bacterial flora isolated prior to cataract surgery. Infect Drug Resist. 2012;5:37-41.

15. Keshav BR, Basu S. Normal conjunctival flora and their antibiotic sensitivity in Omanis undergoing cataract surgery. Oman Ophthalmol. 2012;5:16-8.

16. Arantes TE, Cavalcanti RF, Diniz Mde F, Severo MS, Lins N, Castro CM J. Conjunctival bacterial flora and antibiotic resistance pattern in patients undergoing cataract surgery. Arq Bras Ophtalmol. 2006;69:33-6.

17. Hoshi S, Urabe K. Risk factors for conjunctival bacterial colonization in preoperative cataract patients. Atarashii Ganka (J Eye). 2011;28:1313-9 (in Japanese).

18. Omatsu Y, Miyazaki D, Tominaga $T$, Matsuura $K$, Inoue $Y$. Bacterial flora in the conjunctival sac in eyes before cataract surgery cultured as routine procedure. Rinsho Ganka (Jpn J Clin Ophthalmol). 2014;68:637-43. in Japanese.

19. Eguchi H, Kuwahara T, Miyamoto T, Nakayama-Imaohji H, Ichimura M, Hayashi T, et al. High level fluoroquinolone resistance in ophthalmic clinic isolates belonging to the species corynebacterim macginleyi. J Clin Microbiol. 2008;46:527-32.

20. Owji N, Khalili MR. Normalization of conjunctival flora after dacryocystorhinostomy. Ophthal Plast Reconstr Surg. 2009;25:136-8.

21. Hamatsu Y, Gotoh Y, Tazawa Y. Changes in bacterial flora in the conjunctival sac following lacrimal drainage surgery. Rinsho Ganka (Jp J Clin Ophthalmol). 2003;57:249-52 (in Japanese). 